\title{
Solar Neutron Decay Protons observed on November 7, 2004
}

\author{
Yasushi Muraki $^{a, b^{*}}$ Jose F. Valdés-Galica ${ }^{c}$, Ernesto Ortiz ${ }^{d}$, Yutaka Matsubara ${ }^{a}$, \\ Shoichi Shibata ${ }^{e}$, Takashi Sako $^{f}$, Satoshi Masuda ${ }^{a}$, Shoko Miyake ${ }^{g}$, \\ Munetoshi Tokumaru ${ }^{a}$, Tatsumi Koi ${ }^{e}$, Akitoshi Oshima ${ }^{b, e}$, Takasuke Sakai ${ }^{h}$, \\ Tsuguya Naito ${ }^{i}$, and Pedro Miranda ${ }^{j}$
}

a) Institutitute for Space, Earth and Environment,Nagoya University, Nagoya, Aichi 464-8601, Japan

b) Astronmical Observatory, Chubu University, Kasugai, Aichi, 487-0027, Japan

c) Instituto de Geofisica, UNAM, 04510, Mexico D. F., Mexico

d) Escuela Nacional de Ciencias de la Tierra, UNAM, 04510, Mexico D.F., Mexico

e) Engineering Science Laboratory, Chubu University, Kasugai,Aichi 487-0027, Japan

f) Institute for Cosmic Ray Research, The University of Tokyo, Kashiwa, Chiba 277-8582, Japan

g) Ibaraki National College of Technology, Ibaraki College, Hitachinaka, Ibaraki 312-8508, Japan

h) Physical Science laboratory, Nihon University, Narashino, Chiba 275-0006, Japan

i) Information Science. Labortairy, Yamanashi Gakuin University, Kofu, Yamanashi 400-8575, Japan

j) Instituto de Investigaciones Fisicas,, UMSA, La Paz, Bolivia

E-mail: muraki@isee.nagoya-u.ac.jp

\begin{abstract}
We have found an interesting event registered by the solar neutron telescopes installed at high mountains in Bolivia (5250 m a.s.1.) and Mexico (4600 $\mathrm{m}$ a.s.1.). The event was observed on November 7th of 2004 in association with a large solar flare of magnitude X2.0. Some features in the registers reveal the presence of solar neutrons, but also possible solar neutron decay protons (SNDP). SNDPs were recorded on board ISEE3 satellite in June 3rd, 1982 . On October 19th, 1989, the ground level detectors installed in Goose Bay and Deep River revealed the registration of SNDPs. Therefore this is the second example that such an evidence is registered on the Earth's surface.
\end{abstract}

\section{Introduction}

Gamma rays and neutrons propagate freely in the interplanetary medium when emitted as secondary products of solar explosion events. Therefore they may provide information regarding the condition of production site and mechanism of acceleration. Solar neutrons have been observed in space crafts and ground based detectors [1-4]. In order to push forward

37th International Cosmic Ray Conference (ICRC 2021) July 12th - 23rd, 2021

Online - Berlin, German 
this study, we have an international solar neutron telescope (SNT) network at high mountains around the world. In this paper we report the registers obtained at two Solar Neutron Telescopes installed at high mountains that could be interpreted as products of solar neutrons and solar neutron decay protons (SNDP) produced at the 7 November 2004 X2 solar flare.

\section{The two mountain detectors located on the American continent}

Two solar neutron detectors are located at Mt. Sierra Negra $\left(4,600 \mathrm{~m}, 19.0^{\circ} \mathrm{N} 97.3^{\circ} \mathrm{W}\right)$ and Mt. Chacaltaya $\left(5,250 \mathrm{~m}, 16.3^{\circ} \mathrm{S} 68.1^{\circ} \mathrm{W}\right)$. The detector installed at Mt. Chacaltaya in Bolivia has of $4 \mathrm{~m}^{2}$ of plastic scintillator with $40 \mathrm{~cm}$ thickness. The detector installed at Mt. Sierra Negra, Mexico is composed of $4 \mathrm{~m}^{2}$ plastic scintillator with $30 \mathrm{~cm}$ thickness. In addition, four layers of proportional counters are installed underneath the scintillator for the identification of the arrival direction of charged particles [5]. At the top of the anti-counter, $5 \mathrm{~mm}$ thick lead plate was installed. These SNTs were constructed for the detection of solar neutrons. Both instruments distinguish neutral from charged incident particles.

\section{Signals recorded in the Solar Neutron Telescopes}

Increases of the counting rate were recognized in the 5-minute-value of the solar neutron telescopes (SNT) located in Bolivia from 15:48 to 16:06 UT, and in Mexico from 15:51 to 17:09 UT of November 7th, 2004. The statistical significance of each excesses was $3.7 \sigma$ and $12 \sigma$ respectively. Both data are shown in Figures $\mathbf{1}$ and 2. The red lines in the figures show the neutron emission time at the Sun estimated from GOES X-ray data at 15:47:00 UT.
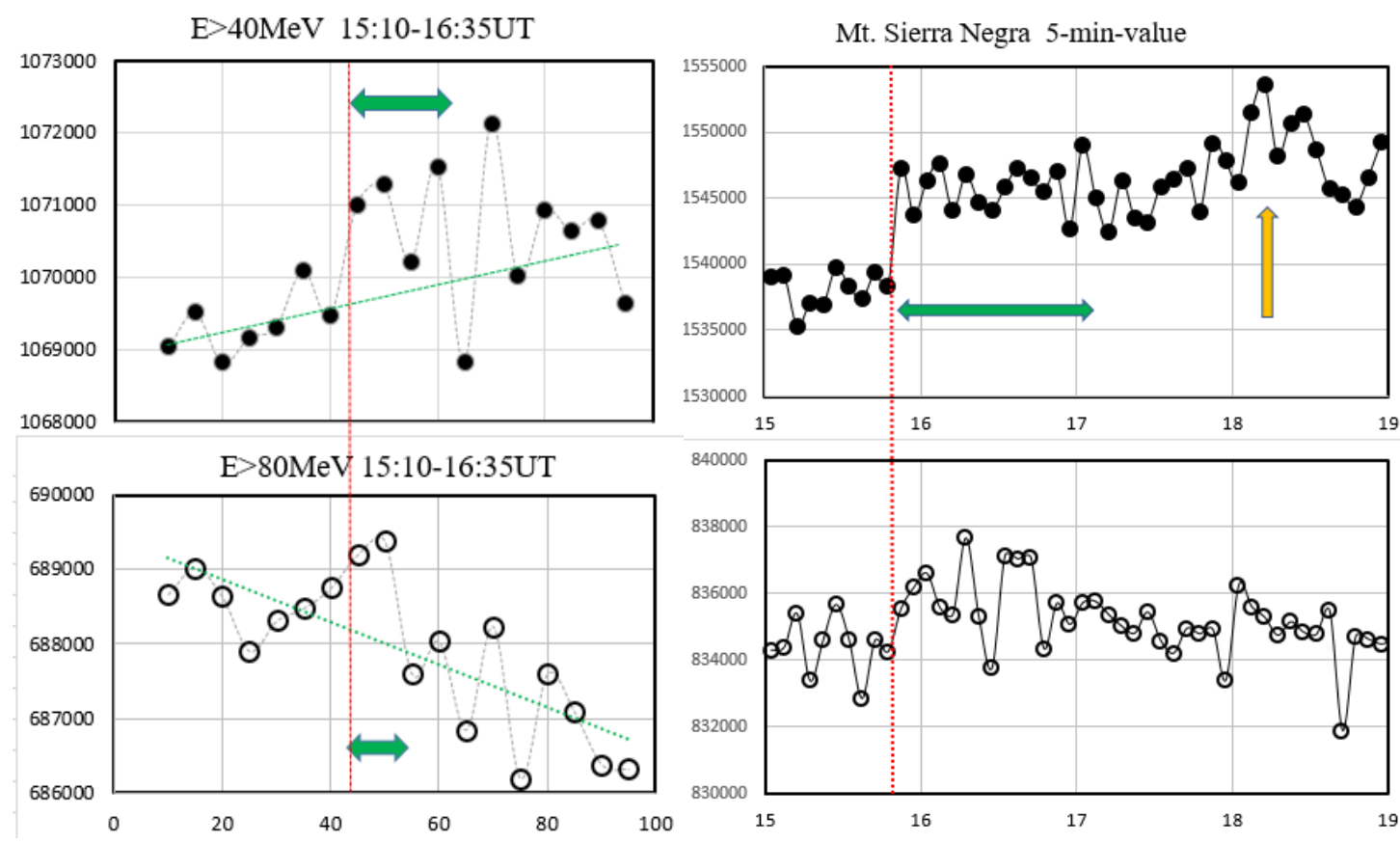

16:00UT

Figure 1. Counting rate of Chacaltaya SNT. Vertical axis presents the 5-minute value of counting rate. The green arrow presents the arrival time of solar neutrons.
Figure 2. (up) Sierra Negra counting rate of anticounter $(>2 \mathrm{MeV}) / 5$ min. (down side) charged particles with $\mathrm{E}>30 \mathrm{MeV}$. Horizontal axis shows the time in UT.

The excesses of signal of the SNTs started almost at the same time, assuming them to be due to the solar flare of X2.0. The increase cannot be explained by fluctuations of the background. 


\section{Interpretations of the events}

We make the following hypotheses in order to explain the observations:

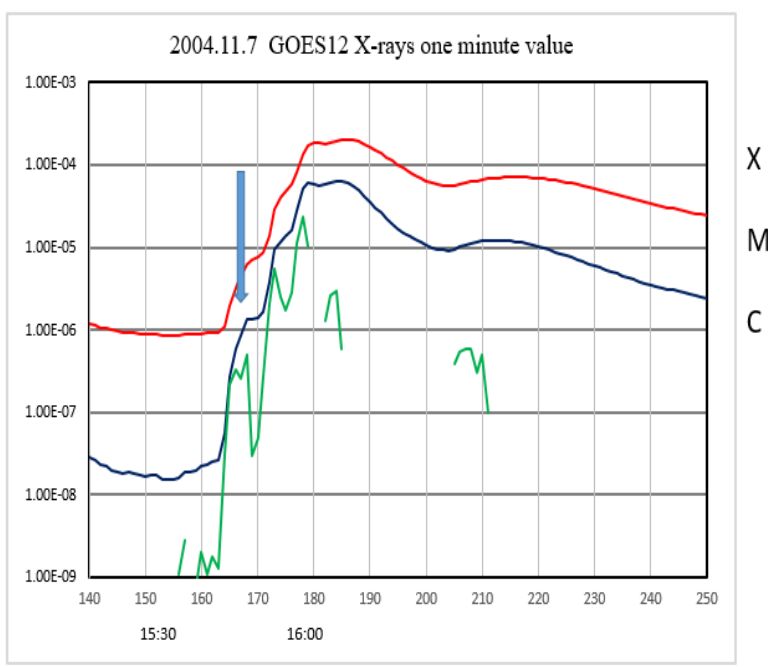

(a) The increase of the 5-minute counting rate registered during 15:48-16:06 UT at Chacaltaya was induced by solar neutrons. These neutrons were produced by the impulsive X2.0 flare. The most probable production time is when the derivative of the GOES X-ray intensity showed the first maximum at 15:47:00 UT as shown by the arrow in Figure 3. There we can see that the flare reached X2.0 via three steps. The tendency is quite similar to the event observed in April 15, 2001 [6].

Figure 3. The GOES X-ray intensity around 15:30 - 16:00 UT. X, M, C represents the class of X-ray intensity.

(b) The increase of the counting rate observed between 15:51 and 17:09 UT at Mt. Sierra Negra was due to solar neutron decay protons (SNDP) produced in the space between the front of the Coronal Mass Ejection (CME) and the Earth. We specify the preceding CME as CME1 (Figure 4). Two large CMEs were produced: one in November 6th at 00:34 UT (CME1) and another in 7th at 16 UT (CME2). Possible acceptable area for the SNDP by each detector is shown in Figure 4. The distance D1 in Figure 4 is estimated to be $10^{7} \mathrm{~km}$. First, the SNDP produced near the magnetosphere arrived (GOES event), then the SNDP produced near the CME1 transported to the Earth (Sierra Negra event). The CME1 shock front arrived the Earth at 18:30UT [7]. The time is indicated by the yellow arrow in Figure 2.

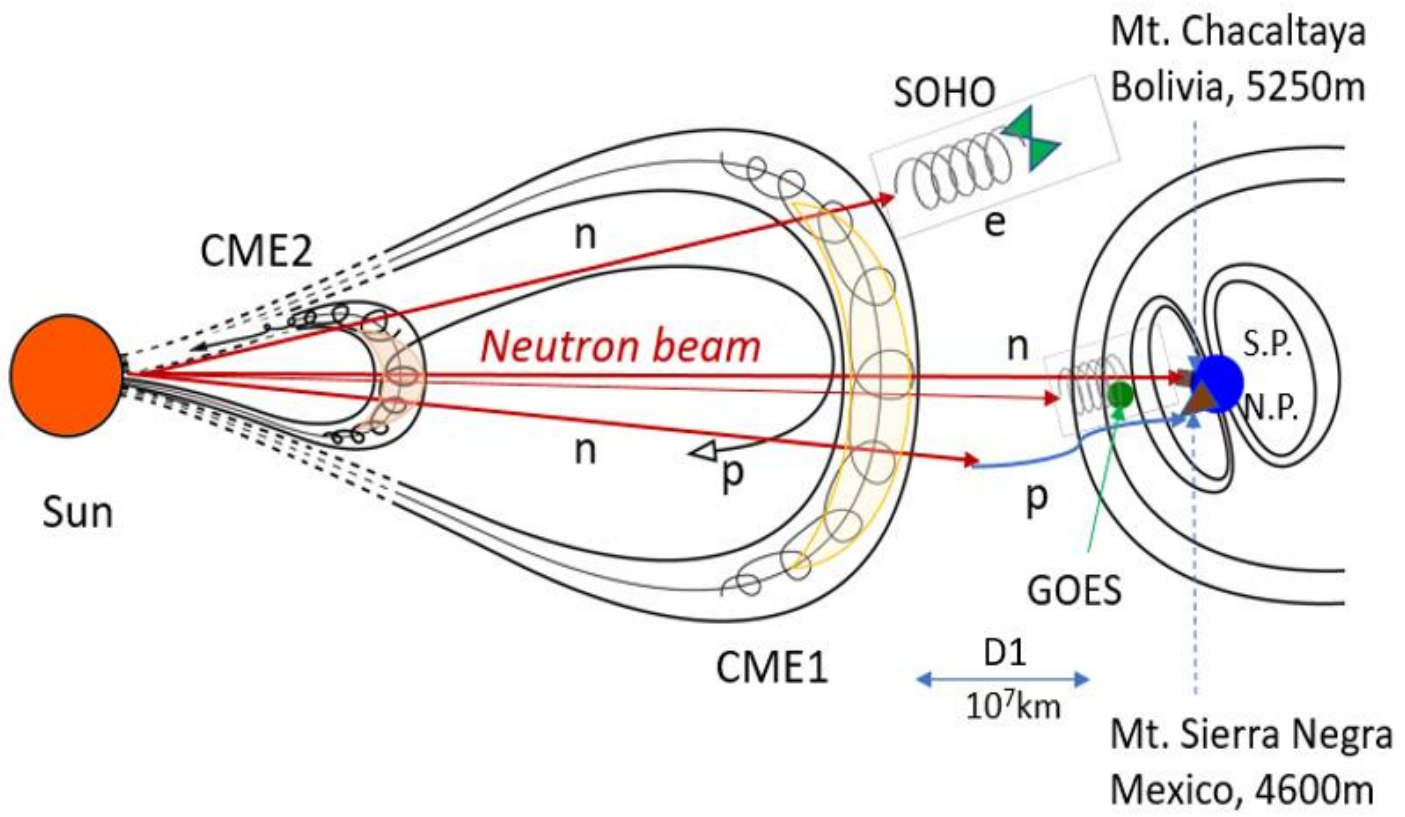

Figure 4. General view of the interplanetary space at the time of the discussed event. 
The hypotheses (a) and (b) were introduced based on two reasons. One arises from the difference of the atmospheric thickness of the neutron path length between the two stations. At the flare time, there were $200 \mathrm{~g} / \mathrm{cm}^{2}$ difference of atmospheric thickness between the two observatories for the passage of neutrons [8-10]. Therefore the flux of neutrons at Mt. Sierra Negra should be 7 times less than that of Chacaltaya ( 1/7), because both detectors are located at nearly the same altitude. (Let us remind that the Sun was situated above South America.) However, both excesses were detected with nearly the same intensity.

The second reason is based on the difference of the event duration. The signal of Chacaltaya was observed during 18 minutes, while the excess of Sierra Negra continued for 78 minutes (as shown by the horizontal green arrow in Figure 2). Neutrons with an energy of $50 \mathrm{MeV}$ are expected to arrive to the Earth 18 minutes later than the fastest neutron, if neutrons were produced instantaneously. It is possible to detect neutrons with energy $50 \mathrm{MeV}$ by the Chacaltaya detector, since the threshold energy is set at $>40 \mathrm{MeV}$. On the other hand, neutrons with an energy of 30 $\mathrm{MeV}$ arrive at the Earth after 22 minutes (1,300 seconds) later than the highest energy neutron. The threshold energy of the S1 channel of the Sierra Negra SNT is set at $>30 \mathrm{MeV}$. However, the increase continued for 78 minutes. Therefore it would be difficult to explain the signals observed at Mt. Sierra Negra by the direct hit of solar neutrons.

The charged particles observed at Mt. Sierra Negra were produced in the interplanetary space and transported toward the Earth being trapped by the magnetic-field. This may be one of the reasons why the excess of Mt. Sierra Negra continued for 78 minutes and was slightly delayed with respect to the fastest neutron arrival time [11]. In comparison with the June 3rd, 1982 event [12], the SNDP of November 7th, 2004 event was short $(\sim 1 / 10)$. One of the reasons may be due to the difference of the decay space of the SNDP between the events.

The excess of the counting rate at Chacaltaya was $\sim 100$ events $/\left(\mathrm{m}^{2} \cdot\right.$ minute $)$ in the $>40 \mathrm{MeV}$ channel between 15:48 UT and 16:06 UT. On the other hand, the S1 channel of Sierra Negra (the channel for charged particles with the energy higher than $>30 \mathrm{MeV}$ ) showed 133 events / $\left(\mathrm{m}^{2} \cdot\right.$ minute $)$ as the excess between 15:51-16:27 UT. The flux was observed with nearly the same intensity as that of Chacaltaya, and the excess start time was nearly the same. However, the excess duration was completely different. Therefore, in order to explain both enhancements consistently, we may introduce the assumption that the excess of Chacaltaya was produced by the direct arrival of solar neutrons, while the excess of Sierra Negra was produced by solar neutron decay protons (SNDP).

c) We also point out that the increase observed by the GOES 11 satellite during 15:50-16:00 UT was produced by neutron decay protons (Solar Geophysical Data 2004) [13]. They were decay products of high energy neutrons in the energy range between 80 and $400 \mathrm{MeV}$. If these neutrons were produced at 15:47 UT, from the observed time, the parent neutrons had the energy between 80 and $400 \mathrm{MeV}$.

Figure 5. The GOES11 X-ray intensity curve. A $16 \sigma$ enhancement was observed.

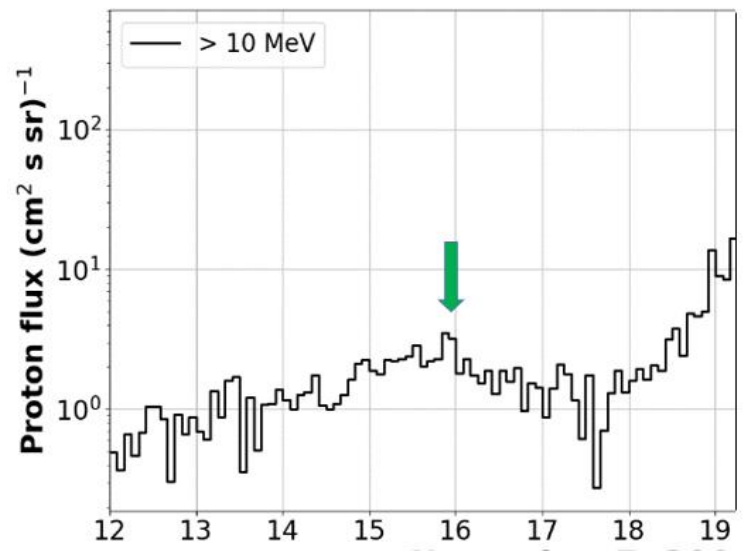




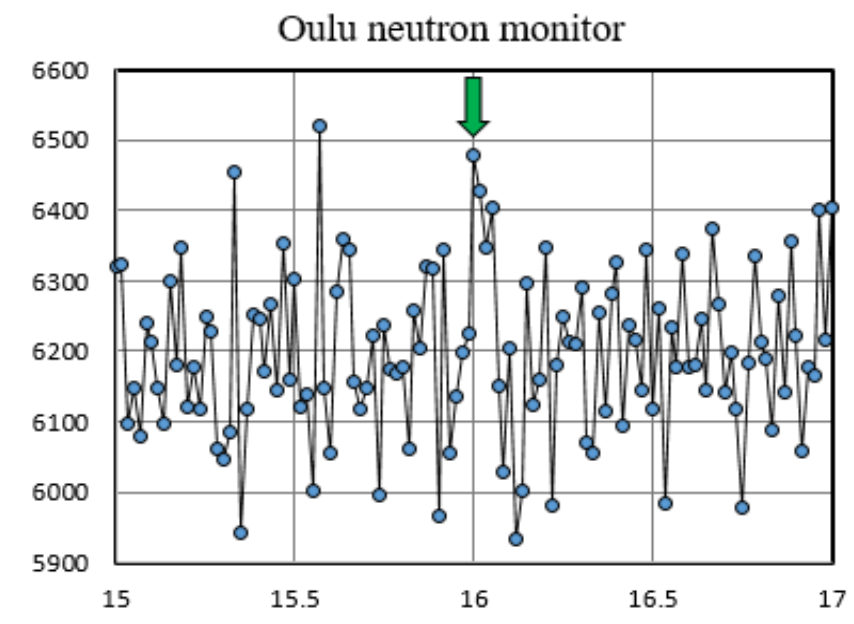

Figure 6. Vertical number presents one-
minute-value of Oulu Neutron Monitor.

(d) We also present the one-minutevalue of the Oulu neutron monitor in Figure 6. During 16:00-16:03 UT (indicated by the arrow), a $5.3 \mathrm{\sigma}$ enhancement is recognized. The cut-off rigidity of Oulu is $0.78 \mathrm{GV}$. On the other hand, the neutron monitors located at the South Pole showed minor excesses. All these could be due to SNDP.

\section{Estimating the flux of SNDP at Mt. Sierra Negra}

To estimate the flux of SNDP, we may take into account another effect, since the energy of the SNDP is expected to be around a few GeV. After transportation of SNDP in the magnetosphere, some of them may be trapped by the Earth's magnetic field, but some of them will penetrate and arrive over Mt. Sierra Negra. The characteristic energy is known as the cutoff energy of primary low energy protons or Störmer limit (rigidity). The rigidity of Mt. Sierra Negra for vertical entrance is estimated as $7.5 \mathrm{GV}$, while for the protons from the west direction it is $5.5 \mathrm{GV}$.

The "gate energies" were calculated by tracing back the anti-proton trajectories ejected upward from Mt. Sierra Negra; whether these anti-protons can arrive at the magnetosphere boundary or not ( 9.5 R_E) [14]. On the other hand, the rigidity at Mt. Chacaltaya was estimated as 11 12 GV [15].

The differential energy spectrum of protons near the rigidity has been measured in space by PAMELA [16] and AMS [17] independently. The result is shown in Figure 7 by the green triangles for the rigidity energy region of 2-8 GV. The expected flux of SNDP near the rigidity at the top of the atmosphere is shown by the open circles $(0)$ in Figure 7. They are made by multiplying the PAMELA's observed differential flux of $2-7.5 \mathrm{GeV}[18,19]$ to the expected neutron energy spectrum of $\mathrm{En}^{-4} \mathrm{dEn}$ (the red diamond in Figure 7). The energy spectrum of SNDP beyond the cut-off energy is expected to follow the neutron energy spectrum. However, in the low energy region, less than the rigidity energy, the energy spectrum of SNDP at the top of the atmosphere is predicted to have nearly constant value to the 0.0001 neutron spectrum of $\mathrm{En}^{-4} \mathrm{dEn}$. The expected flux must also take into account the attenuation of secondaries in the atmosphere. They are shown by the full circles $(\bullet)$ in Fig 7 . The effect has been estimated by the GEANT4.

Expected diffrerential energy spectrum of SNDP

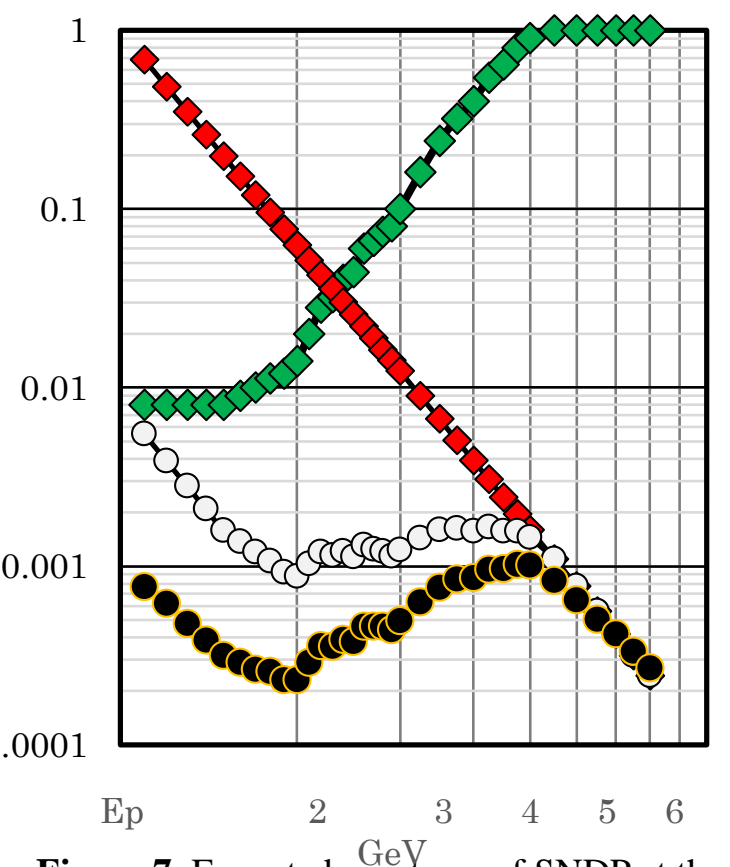

Figure 7. Expected spectrum of SNDP at the top of the atmosphere (o) and Mt. Sierra Negra $(\bullet)$. 


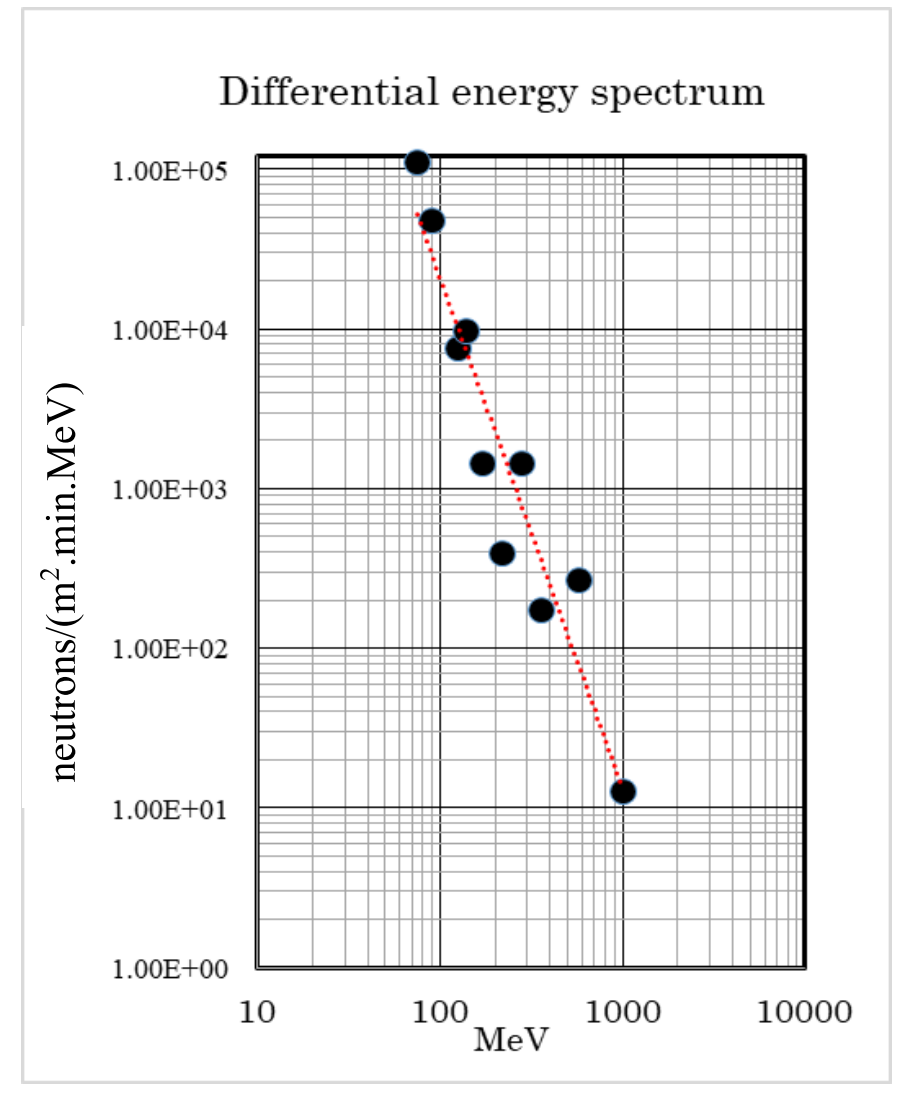

Figure 8. The differential energy spectrum of solar neutrons observed in Bolivia on November 7, 2004.

In order to derive the differential energy spectrum, we introduce the hypothesis that neutrons were produced impulsively at the Sun. The fixed fixed production time is at 15:47:00 UT.

Then the kinetic energy of neutrons is uniquely determined from the flight time. Then the differential flux of solar neutrons is derived as a function of energy after correction of the detection efficiency [20] and the attenuation in the atmosphere [21].

Figure 8 provides the flux at the top of the atmosphere and the flux is normalized to the unit of area $\left(/ \mathrm{m}^{2}\right)$.

\section{Comparison with the fluxes}

\section{a) Deriving solar neutron flux at the top of atmosphere over Chacaltaya.}

At 16 UT (12 Local Time), the atmospheric thickness over Chacaltaya is estimated as $550 \mathrm{~g} / \mathrm{cm}^{2}$. The differential energy spectrum is presented in Figure 8. Figure 8 is derived based on the oneminute value of the counting rate.

\section{b) Flux of solar neutron decay protons over Mt. Sierra Negra}

Next we will estimate the flux of SNDP at Mt. Sierra Negra. We use the event rate recorded by the Mt. Sierra Negra SNT in between 15:51 UT and 15:54 UT. They may carry information on the flux near the rigidity energy region of protons around E_cut $\sim 5.5 \mathrm{GeV}$. Such high energy protons make nuclear interactions with air nuclei and produce small size air showers. The process has been simulated by GEANT 4. The results are shown in Figure 9, where it is shown that the flux of secondary particles (gamma-rays $(\bullet)$ and neutrons $(\bullet)$ with the energy higher than E $>30$ $\mathrm{MeV}$ ) has almost keep the intensity of the incident proton flux of $\mathrm{E}_{\mathrm{p}} \sim 5.5 \mathrm{GeV}$.

Figure 7 suggests that the SNDP between the energy range $\mathrm{Ep}=3.5-6 \mathrm{GeV}$ may be observed at Mt. Sierra Negra. Therefore, we choose the width of the energy bin $(\Delta \mathrm{E})$ as $2500 \mathrm{MeV}(=6000$ $3500 \mathrm{MeV}$ ) and divide the observed intensity by $\Delta \mathrm{E}$. Then the differential flux of the SNDP in $3.5-6 \mathrm{GeV}$ (at the top of the atmosphere) is estimated as $(0.23 \pm 0.02) /\left(\mathrm{m}^{2} . \mathrm{min} . \mathrm{MeV}\right)$. On the other hand the flux of solar neutrons at the top of the atmosphere over Mt. Chacaltaya is estimated as $12.8,1.8,0.5,0.2$ and 0.1 events $/\left(\mathrm{m}^{2}\right.$.min.MeV) at $\mathrm{En}=1,2,3,4$ and $5 \mathrm{GeV}$ respectively. (We extended the red line of Figure 8 up to $5 \mathrm{GeV}$.) If we compare these fluxes, the estimated flux of SNDP at Mt. Sierra Negra (0.23) was just twice higher than the Chacaltaya neutron flux (0.1) at $\sim 5 \mathrm{GeV}$. Taking into account the decay probability of $\mathrm{E}_{\mathrm{n}} \sim 5 \mathrm{GeV}$ neutrons of $(\sim 0.0077(\sim 1 / 130))$, 
the value is too high. We may find out a solution to resolve the difference in a point of acceptance difference between protons and neutrons between protons and neutrons $\left(4 \mathrm{~m}^{2}\right)$.

Protons may enter from various area of the magneto-sheath. If the effective acceptance is wide as $1,040 \mathrm{~m}^{2} \quad\left(=32 \times 32 \mathrm{~m}^{2}\right)$, we can explain each flux. There seem to be several routes to the Earth for the SNDPs. It is called cosmic ray penumbra [22]. Here we assume that $\sim 5 \mathrm{GeV}$ neutrons decay in the D1 space of $10^{7} \mathrm{~km}$ of Figure 4.

Figure 9. The inter-gap at $570 \mathrm{~g} / \mathrm{cm}^{2}$ is due to the lead plate.

\section{Discussions}

The possibility of a chance coincide between the arrival of high energy protons accelerated by the CME2 and solar neutrons exists. However the magnetic field of the CME1 (40nT) may prevent entering the accelerated particles with an energy less than $60 \mathrm{GeV}$ into the space between the CME1 and the Earth.

Here we point out a result of GEANT4. The simulation suggests that the ratio between the anticounter and the $\mathrm{S} 1$ channel to be 0.5 at $1 \mathrm{GeV}$. However the ratio increases from 1.25 to $1.7 \sim 2.0$ from $\mathrm{Ep}=4$ to $5.5 \mathrm{GeV}$. Furthermore it predicts 2.0 at $7 \mathrm{GeV}$. The result of the observation is $(2.0 \pm 0.7)$ and this is consistent with our assumption that the excess was made by protons with energies around $5 \sim 7 \mathrm{GeV}$.

\section{Concluding Remarks}

We have presented evidences based on two ground based detectors. Taken together, observations admit an interpretation that the excesses would explained by the observation of solar neutrons and SNDP. Solar neutron decay protons were first reported in 1981 by Evenson et al. [23]. Ruffolo and other researchers discussed solar neutron decay electrons and protons [24, 25]. Another possible candidate of SNDP has been found in the GOES-7 proton data of 1990 May 24 (Solar Geophysical Data sgd9006.pdf [26] and in [27, 28].

However, we do not know any report of this kind of events after that. To the best of our knowledge, there are only four early reports on the detection of neutron decay protons and electrons onboard satellites and one report of neutron decay protons by ground level detectors in October 19th, 1989 [27] The event of October 1989, was also discussed by Koi et al. [29]. So, the event presented here may be the second case where neutron decay protons were registered by a ground-based detector. Further study is necessary to understand this phenomenon deeply. However, we report this fact to call community attention in the search of neutron decay protons and electrons in solar events.

\section{Acknowledgments}

The authors acknowledge INAOE personnel and authorities for their continued support in providing the services needed to keep the Mt. Sierra Negra SNT functioning. The authors express the acknowledgment to the staffs of physics department of UMSA, who have kept the Solar 
Neutron Detector located at Mt. Chacaltaya under good condition for 28 years. This work has been carried out, being based on the international solar neutron telescope (SONTEL) network data reserved in the storage system of ISEE, Nagoya University. The authors acknowledge Prof. Philip Yock of Auckland University to provide useful comments.

\section{References}

[1] Valdés-Galicia et al. (2009) NIMA 43: 565-572.

[2] Dorman L. (2010) Solar Neutrons and Related Phenomena (Springer) pp374-377.

[3] Kamiya K. et al. (2019) PoS (ICRC2019) 1150 and PASJ (to be published in 2021).

[4] Muraki Y. et al. (2020) Pub; Astron. Soc. of Japan 72:1-17.

[5] Valdés-Galicia et al. (2004) NIMA 535: 656-664.

[6] Muraki Y. et al. (2020) Astroparticle Physics 29:229-242.

[7] Tokumaru M. (2013) Proceed. of Japan Academy Serb B, 89(2):67-79.

[8] Dorman L et al. (1997), (1999) JGR 104 (A10):22417-22426.

[9] Tsuchiya H. et al. (1999) Proceed $26^{\text {th }}$ ICRC (Salt Lake City) 7:363.

[10] Tsuchiya H. et al. (2001) Proceed 27th ICRC (Hamburg) 8: 3056-3059.

[11] Sakai T. et al. (1997) Earth. Geomag. Geoelectric. 49:1105-1113.

[12] Evenson P. et al. (1983) ApJ 464:L87-90.

[13] Solar Geophysical Data web site: https://www.ngdc.noaa.gov/stp/space-weathrt/ online-publications/stp sgd/1982/sgd8212c.pdf

[14] Miyake S. et al. (2017) Space Weather 15:589-605.

[15] Shea M.A. and Smart D.F. (2000) Space Science Review 93:187-205.

[16] PAMELA collaboration: De Simone et al. (2009) Proceed. $31^{\text {st }}$ ICRC (Lodz) icrc0795. Adriani et al. (2016) Space Weather 14: 210-220.

[17] AMS collaboration: Alearaz et al. (2000) Physics Letters B472:215-226.

[18] Casolino M. et al. (2007) J. Phys. Soc. Japan. Suppl. A78:35-40, also Casolino et al. Proceed. 30 ${ }^{\text {th }}$ ICRC (Merida) 1: 709-712.

[19] Flückiger E.O. (2007) Proceed 30 ${ }^{\text {th }}$ ICRC (Merida) 6: 239-253 (Rapporteur talk).

[20] Watanabe K. (2005) Proceeding of Cosmic-ray Research section of Nagoya University 46(2), pp1-249. PhD thesis to Nagoya University (in English).

[21] Shibata S. (1994) JGR 99:6651-6665.

[22] Smart D.F. Shea M.A. and Flückiger E.O. (2000) Space Science review 93:305-333.

[23] Evenson P., Kroger R., Meyer P. and Reames D. (1990) ApJ Suppl. 73:273-277.

[24] Ruffolo D (1991) ApJ 388: 688-698.

[25] Dröge W., Ruffolo D., and Klecker B. (1996) ApJ 464:L87-L90.

[26] Solar Geophysical Data web site: sgd9011c and sgd9006p www.ngdc.noaa.gov/stp/space-weather/online-publications/stp_sgd/1990/

[27] Shea M.A., Smart D.F., Wilson M.D., and Flückiger E.O. (1991) GRL 18: 829-832.

[28] Kocharov L.G., Kovaltsov G.A., Torsti J. and Vanio R. (1995) Proceed. $24^{\text {th }}$ ICRC (Rome) 4: 163-165.

[29] Koi T. et al. (1993) Proceed. of 23 $3^{\text {rd }}$ ICRC (Calgary) 3:151-154. 